\title{
Fashion communication research: A way ahead
}

\author{
Lorenzo Cantoni, USI - Università della Svizzera italiana, Switzerland \\ Francesca Cominelli, Université Paris 1 Panthéon-Sorbonne, France \\ Nadzeya Kalbaska*, USI - Università della Svizzera italiana, Switzerland \\ Michela Ornati, USI - Università della Svizzera italiana, Switzerland \\ Teresa Sádaba, ISEM Fashion Business School, Spain \\ Patricia SanMiguel, ISEM Fashion Business School, Spain \\ ${ }^{*}$ Corresponding author: nadzeya.kalbaska@usi.ch
}

\begin{abstract}
This document, designed and written during the conference FACTUM19, has two main goals: to help consolidate Fashion Communication as an academic field, and to support an international and interdisciplinary network of scholars in the area, whose collaboration may lead to a larger body of research and, in turn, to further academic recognition. To do so, it outlines main research topics, approaches and challenges, as well as related industry issues and learning dimensions.
\end{abstract}

\section{Keywords}

fashion communication, fashion, digital fashion, communication research

Fashion is a dynamic field in constant evolution. It impacts people and businesses, but also culture, art, and the whole ecosystem, embracing beauty, gastronomy, decoration, home wear, music and tourism ... In a word - lifestyles.

Fashion is (also) communication: the way we dress is a major channel through which we express ourselves and communicate our identity to others. Moreover, Fashion Communication gathers many communication professionals and fields, e.g.: journalism, corporate communication, internal communication, external communication and marketing, public relations, crisis communication, social media, and many others.

The digital transformation is deeply impacting fashion and its communication - Fashion media logic - as it is impacting the way we live, think, and collaborate. Digital fashion is pushing fashion brands to become also media companies, hence to further stress their communication role.

However, research on Fashion Communication has not received major attention by communication scholars and institutions, and those studying it do not form yet a fully recognizable research community ...

\section{How this document has been designed and developed}

In the framework of "FACTUM19-Fashion Communication Conference", an ad-hoc workshop has been held and organized as follows.

A first meeting took place on July $22^{\text {nd }}$, 2019. Ideas have been crowdsourced from all participants (about twenty-five) on four main dimensions: (a) research topics; (b) research methods and limitations; (c) collaborations with the industry; and (d) teaching / learning activities. Later on, groups were set-up and worked to organize the proposed ideas, and to ensure consistency and completeness of the resulting landscape. Every group had a rapporteur, in charge of leading the conversation and of documenting its results.

On July $25^{\text {th }}$, a second meeting was held. Rapporteurs presented their group a first draft document, and improved it thanks to group feedback. Their activity leveraged also on a preparatory work, which analyzed published papers on the topic presented at relevant events or in dedicated journal issues tackling Fashion Communication. The FACTUM19 Conference Proceedings have been also analyzed: 
most frequent keywords were studied to ensure a wide coverage of current studies and research trends.

The document has been further improved and refined by rapporteurs. Finally, participants have been sent the final version and invited to sign it.

\section{Results}

Hereafter the results, organized according to the four above-listed main areas.

\subsection{Key research topics and emerging trends}

Due to the complexity of the studied object - the concerned human experiences and industry practices - the topics studied in Fashion Communication involve many disciplines.

The following tracks are suggested as crucial to be covered by the Fashion Communication research community.

\section{Essentials in communication and media}

Among them: communication practices and strategies concerning information sharing, the relationships with all involved stakeholders, with different generations and cultures. Regarding communication channels, it is still necessary to analyze their differences and relationships, and how they are being re-negotiated under the digital transformation. It is also interesting to focus on: media power, media education, media hybridization and the role of fashion journalism within journalism at large.

Further focus should be on the characteristics of the fashion language, which involves all our senses, using textual elements as well as images, videos, sounds. These multimedia practices have been allowed and promoted by the emergence of digital communication.

\section{Digital Fashion Communication}

In the digital age, communication and marketing have become even more interrelated. Therefore, this track analyzes both aspects. It is important to underline that the digital fashion ecosystem is in con- stant evolution, and multi / cross / omni channel processes are to be analyzed.

Social media platforms have been established as major channels to connect the different fashion stakeholders. From a communication perspective, it is needed to understand message creation, community and network dynamics, the power of electronic word-of-mouth, relation and impact of traditional and emerging actors - such as celebrities, opinion leaders, bloggers and digital influencers.

Online communication is also a way to develop digital marketing strategies, involving advertising, analytics, artificial intelligence, mobile apps, instore and eCommerce technologies, personalization and localization practices, chatbots, augmented and virtual reality, recommending technologies and many others. Also, human-computer interactions studies are needed here, when it comes to fashion-related applications, focused on user experiences and usage practices.

\section{Fashion users/consumers}

Fashion Communication constantly influences and impacts on fashion users and consumers. In this way, research concerning perception and self-perception, motivation, attitude, behavior, satisfaction, and loyalty are still needed, keeping into account the digital transformation.

To analyze the messages and communication exchanges that occur during a customer's journey is also crucial, because it has an impact on the buying behavior and on brands - equity, experience, identification, relationship.

\section{Fashion brands}

The way each brand communicates its identity, values and purpose is a key issue for researchers as well as for practitioners. Many fashion brands aim to communicate their heritage either intangible or tangible, as it helps to consolidate their reputation and prestige. The path companies choose to increase and engage their audience is often pursued through an attractive storytelling and the promotion of new perspectives and imaginaries, which need to be carefully studied. 
New developments in this field are having more and more importance, as brands are creating their own communication products like fashion shows, fashion films, brand magazines, etc. In the same way, fashion brands also communicate through their product, packaging and visual merchandising.

The co-creation of brand identity by fashion companies and user-generated contents is a major topic of research, which pushes also to address the issues of sustainability and ethics, which are more and more at the center of fashion-related discourses.

\section{Sustainability and ethics}

Fashion Communication is being affected by a revolution related to sustainability and ethical issues, whose communication deserves a major research attention, also for its direct impact on brand reputation.

Ethical behaviors include the need to consider environmental aspects, such as climate change, and social aspects - morality, exploitation, cultural appropriation, globalization...

In fact, several times the (problematic) relations between fashion and sustainability and ethics emerge in crises - indicating that crisis communication has to be taken particularly into consideration by researchers. In addition, studying legal aspects, privacy and copyright issues that influence Fashion Communication is essential.

\section{Culture and Identity}

Fashion Communication is directly impacted by many cultural and identity aspects.

The emerging interest about fashion by museums and other main cultural players is a further incentive for Fashion Communication researchers to understand the importance of their studied domain not only on the ephemeral market of everyday marketing and communication messages, but also on the major long-term symbolic exchanges that shape cultures and societies.

Among the main issues to be researched: intercultural communications, territory as inspiration for fashion indus- try, fashion and cultural heritage, cultural appropriation, culture in the use of social media, identity of marginalized people. Additionally, more research attention is needed when it comes to the Global South and less affluent societies and communities.

\subsection{Research methods and their limitations}

As stressed in the Foreword, methodology in the Fashion Communication field should promote interdisciplinarity, through theoretical and empirical works. Research cannot be limited to a fashion object, brand, or discourse: it should aim to deal with complex and evolving systems through contextual analysis.

Case studies are crucial to understand the dynamics of Fashion Communication, to ground and validate theoretical frameworks, to allow for comparisons at different levels: local / global, personal / collective, short / long term. Case study analysis needs to be based on a clear methodology and fundamental references to collect and analyze data.

Qualitative methods - e.g.: interviews, focus groups, ethnographic research, passive and active observations, textual and semiotic analysis - do constantly interact with quantitative methods - e.g.: questionnaires, statistical analyses, digital analytics. These methods should take into consideration the new findings in each field, e.g.: automatic image recognition, eye tracking and other biometrical data, automatic language processing, sentiment and argumentation analysis, neurosciences, and so on.

Research processes need to be supported by consistent publications on distinct academic publication outlets, sometimes still missing in this domain.

The sharing, ideally using open content licenses, of research outcomes and data is crucial to improve the research in this field and to promote wider collaborations and understanding of research results. Innovation in research might rise from the creation of labs, physical or virtual, gathering together thought leaders from academy, industry, public institu- 
tions, culture, science, as well as from other domains.

Finally, research in Fashion Communication from the definition of research hypothesis, through the collection and analysis of qualitative and quantitative data, till the dissemination of its results, should be built on and follow clear and explicit ethical principles.

\subsection{Collaborations between research and industry}

Scholars in Fashion Communication interested in collaborating with the industry fashion firms and media - face a number of issues, underscored by what might be a fundamental misunderstanding between both parties. To address those issues, it seems necessary to (i) further define an industry / field in constant change; (ii) clarify the role of communication at different levels in the fashion domain; (iii) "help" companies understand the importance of academic research; (vi) identify companies and organizations interested in such research; and (v) facilitate the negotiation of (less restrictive) Non-Disclosure Agreements.

Collaboration in Fashion Communication research should entail a mutually beneficial strategy: academia should seek to further embed itself into the industry as well as to draw practitioners into its world. This strategy should be implemented via research and development activities, industry-relevant educational programs, and cross-disciplinary initiatives.

It is to be stressed that ethical issues should be constantly considered. Among them, research freedom, privacy and authorization by involved studied persons, careful treatment of data sources, and so on.

\subsection{Research and learning / teaching practices}

While developing teaching and learning activities in Fashion Communication, the use of critical and reflective methodologies is recommended, as well as a longterm approach, which situates the current digital communication environment within the history of Fashion Communication, its practices and its media.
Higher education requires a medium- or long-term approach, hence it is not enough to follow current industry needs, we need to even anticipate them: to do so, extensive and advanced research is a necessary pre-requisite for adequate curriculum and course planning.

In order to be able to communicate fashion, learners should take into consideration every stakeholder: we need to "walk the talk" and be able to listen and to recognize all professional roles in their authenticity.

It is important to create and develop communities for Fashion Communication teachers and learners, also through digital social networks, so to help them networking and learning from each other.

While teaching relevant topics, it is necessary to promote soft skills, which students need to have in order to enter and successfully remain in the job market. Those are creative and critical thinking, writing skills, public speaking, visual literacy, as well as (inter)cultural sensitivity and competency.

When it comes to the modality of teaching and learning, collaborative and creative exercises are recommended, along with group projects, which might help learners to enhance their critical thinking abilities as well as to improve their communication skills. Moreover, projects that involve, together with learners of fashion communication, learners of other related domains (e.g.: fashion design, journalism, management, IT, etc.) can be useful to make them better understand the reasoning of other professionals and the approach by different involved disciplines.

Finally, learning and teaching Fashion Communication can benefit from emerging technological solutions, from blended learning to the use of online tutorials and augmented reality applications, up to the creation of MOOCs (Massive Open Online Courses) on the topics related to Fashion Communication, which might benefit higher education students as well as professionals already in the field. 


\section{Conclusions}

This document, quasi a Monte Verità Manifesto on Fashion Communication Research, is the product of the fruitful cooperation among colleagues from different research and cultural backgrounds, yet sharing common ideas and beliefs on the current state and challenges to further establish a research community on Fashion Communication.

Its contents are incomplete and require constant refinement and updates, as needed by such an evolving and exciting research area. It is not intended to indicate limits or to draw boundaries. Rather, it aims to mark a step, as we believe, in the right direction in order to support an international and interdisciplinary network of scholars in this field, and to get further academic visibility.

Monte Verità (Ascona, Switzerland), July 2019

\section{Other signatories who participated in} FACTUM19 (in alphabetical order):

Stefano Airoldi (STA Scuola Tecnici Abbigliamento, Lugano, Switzerland), Romana Andò (Sapienza University, Rome, Italy), Dimple Bahl (National Institute of Fashion Technology, New Delhi, India), Silvia Blas Riesgo (University of Navarra, Pamplona, Spain), Dorrit Bøilerehauge (Aarhus University, Aarhus, Denmark), Elisa Borboni (Politecnico di Milano, Milano, Italy), Simonetta Buffo (Università Cattolica del Sacro Cuore, Milano, Italy), Daniela Candeloro (Sapienza Universi- ty, Rome, Italy), Zhimin Chen (Manchester Metropolitan University, Manchester, UK), Kyung-Hee Choi (Hansung University, Seoul, South Korea), Raluca Creanga (University of Bucharest, Bucharest, Romania), Tiago de Sousa Araújo (Hongik University, Seoul, South Korea), Rossana Gaddi (Politecnico di Milano, Milano, Italy), David Hall (Hongik University, Seoul, South Korea), Hilde Heim (Queensland University of Technology, Brisbane, Australia), Girija Jha (National Institute of Fashion Technology, New Delhi, India), Olga Karamalak (National Research University Higher School of Economics, Moscow, Russia), Katrien Laenen (KU Leuven, Leuven, Belgium), Myles Ethan Lascity (Southern Methodist University, Dallas, Texas, USA), Lisa D. Lenoir (University of Missouri, Columbia, USA), Caroline Lloyd (University of Northampton, Northampton, UK), Anna Fabienne Makhoul (Johannes Gutenberg University, Mainz, Germany), Manish Nangia (National Institute of Fashion Technology, New Delhi, India), Veronica Neri (Università di Pisa, Pisa, Italy), Tekila Harley Nobile (USI, Lugano, Switzerland), Alice Noris (USI, Lugano, Switzerland), Puspita Ayu Permatasari (USI, Lugano, Switzerland), Katharina Sand (Parsons Paris/The New School, Paris, France), Friedemann Schaber (University of Northampton, Northampton, UK), Thomaï Serdari (Leonard N. Stern School of Business, New York University, New York, USA), Jochen Strähle (Reutlingen University, Reutlingen, Germany), Cecilia Winterhalter (Accademia Costume \& Moda, Roma, Italia) 


